\title{
VESTIBOLOGY
}

\section{Defining current practice patterns of vestibular schwannoma management in Italy: results of a nationwide survey}

\author{
L'attuale gestione dello schwannoma vestibolare in Italia: risultati di una survey nazionale
}

\begin{abstract}
Nicola Quaranta ${ }^{1}$, Marco Pontrelli', Sabino Ciprelli', Francesco Signorelli ${ }^{2}$, Luca Denaro ${ }^{3}$, Domenico d'Avella ${ }^{3}$, Giovanni Danesi ${ }^{4}$, Diego Cazzador ${ }^{5,6^{*}}$, Elisabetta Zanoletti ${ }^{*}$

${ }^{1}$ Unit of Otolaryngology, Department of BMS, Neuroscience and Sensory Organs, University of Bari, Italy; ${ }^{2}$ Unit of Neurosurgery, Department of BMS, Neuroscience and Sensory Organs, University of Bari, Italy; ${ }^{3}$ Academic Neurosurgery, Department of Neuroscience - DNS, Padova University, Padova, Italy; ${ }^{4}$ ENT and Skull-Base Surgery Department, Department of Neurosciences, Ospedale Papa Giovanni XXIII, Bergamo, Italy; ${ }^{5}$ Otolaryngology Section, Department of Neuroscience - DNS, Padova University, Padova, Italy; ' Section of Human Anatomy, Department of Neuroscience - DNS, Padova University, Padova, Italy

${ }^{*}$ D. Cazzador and E. Zanoletti contributed equally to this work.
\end{abstract}

\section{SUMMARY}

Objective. Despite the increasing incidence rate of vestibular schwannomas (VS), controversies in their management are still present.

Methods. A 35-item multiple-choice survey investigating the current practice patterns of VS care was sent to the members of the Italian Society of Otolaryngology, Head and Neck Surgery (SIO) and of the Italian Society of Neurosurgery (SINCH).

Results. Among 66 respondents, 37 (56.0\%) claimed to be actively involved in VS management. Most interviewees $(35.1 \%$ ) declared $>20$ years of experience and $59.5 \%$ claimed to work in an academic practice. The number of cases evaluated in each centre per year varied widely, with $54.0 \%$ evaluating $>25$ cases/year and only $13.6 \%>100$ cases/year. Multidisciplinary care for VS evaluation was confirmed by $50.0 \%$ of respondents, and multidisciplinary surgical care by $62.2 \%$. Observation and surgery were the most common management options proposed. Further details regarding VS care are presented.

Conclusions. The present study provides the first overview on the current practice patterns of VS care in Italy. Although integrated in most centres, a multidisciplinary model of care needs to be encouraged. Wide heterogeneity in experience and practices is mostly influenced by the surgeon's different specialties and by the lack of shared guidelines.

KEY WORDS: vestibular schwannoma, acoustic neuroma, microsurgery, stereotactic radiotherapy, skull base surgery

\section{RIASSUNTO}

Obiettivi. Nonostante il costante incremento di incidenza dello schwannoma vestibolare (VS), non vi è ancora uniformità nella gestione di tale patologia.

Metodi. Un questionario di 35 domande sulle strategie di trattamento del VS è stato sottoposto ai membri della SIO e della SINCH.

Risultati. Tra i 66 intervistati che hanno risposto al questionario, il 56,0\% ha confermato di prendere parte attivamente nel trattamento dello VS. La maggioranza dei partecipanti $(35,1 \%)$ dichiarava $>20$ anni di esperienza nel settore, e il 59,5\% di lavorare in un contesto accademico. Il 54,0\% degli intervistati ha affermato di valutare > 25 casi/anno, mentre solo il 13,6\% > 100 casi/anno. Il 50,0\% ha dichiarato di valutare lo VS nell'ambito di un gruppo multidisciplinare, mentre il 62,2\% di operare in un contesto multidisciplinare. L'approccio conservativo e la chirurgia si confermavano le strategie terapeutiche più frequentemente proposte.

Conclusioni. Lo studio presenta una prima panoramica sulle strategie di trattamento dello VS in Italia. Nonostante un modello multidisciplinare di gestione dello VS sia già diffuso in molti centri, è necessario incrementarne ulteriormente lo sviluppo. Le diverse specializzazioni del chirurgo e la mancanza di linee guida condivise contribuiscono a determinare la vasta eterogeneità osservata nella gestione del VS nel nostro Paese.

PAROLE CHIAVE: schwannoma vestibolare, neurinoma dell'acustico, microchirurgia, radioterapia stereotassica, chirurgia del basicranio
Received: September 5, 2020

Accepted: October 13, 2020

\section{Correspondence \\ Diego Cazzador \\ Otolaryngology Section, Department of Neuro- science - DNS, Padova University, via Giustiniani 2, 35128 Padova, Italy \\ Tel. +39049 8218778. Fax +390498211994 \\ E-mail: diego.cazzador@unipd.it}

Funding

None.

Conflict of interest

The Authors declare no conflict of interest.

\begin{abstract}
How to cite this article: Quaranta N, Pontrelli M, Ciprelli S, et al. Defining current practice patterns of vestibular schwannoma management in Italy: results of a nationwide survey. Acta Otorhinolaryngol Ital 2021;41:185-191. https:// doi.org/10.14639/0392-100X-N1107
\end{abstract}

(c) Società Italiana di Otorinolaringoiatria e Chirurgia Cervico-Facciale

\section{(c) (1) $(2)$}

This is an open access article distributed in accordance with the CC-BY-NC-ND (Creative Commons Attribution-NonCommercial-NoDerivatives 4.0 International) license. The article can be used by giving appropriate credit and mentioning the license, but only for non-commercial purposes and only in the original version. For further information: https:// creativecommons.org/licenses/by-nc-nd/4.0/deed.en 


\section{Introduction}

Vestibular schwannoma (VS) is a benign tumour arising from Schwann cells of the VIII cranial nerve and account for $5 \%$ to $9 \%$ of all brain neoplasms ${ }^{1}$. The first evidence in the literature dates to $1777^{2}$. About $90 \%$ of these tumours are unilateral and sporadic. They can also occur bilaterally, generally when associated with genetic conditions such as neurofibromatosis type $2^{3}$. By exerting a compression on the VIII cranial nerve, VS leads to progressive unilateral sensorineural hearing loss (SNHL) and tinnitus in more than $90 \%$ of patients, while vestibular symptoms are present in $\leq 20 \%$. Sudden SNHL may also be the first clinical presentation in up to $22 \%$ of cases ${ }^{4}$.

Over the past 40 years, the incidence rate of VS has steadily increased from approximately 3 cases/million/year to 34 cases/million/year ${ }^{5}$. This steep rise was probably due to VS early diagnosis with high-resolution contrast-enhanced MRI in patients with unilateral or asymmetric audiological symptoms, together with incidental findings from imaging performed for unrelated complaints ${ }^{6}$.

Although several cornerstones of diagnosis and therapy are recognised and shared between different centres, there are still controversies mostly related to the characteristics of tumours and patients, as well as institutional preferences. A major role is probably played by the level of global expertise and the presence of a team. Daveau et al. ${ }^{7}$ reported that, depending on the specialist to whom the patient is first addressed to, the treatment varies significantly, ranging from observation to surgery and radiation therapy. The management of VS is thus strongly influenced by some non-objective parameters, which play a relevant role in the choice of first treatment.

The aim of the present study was to investigate the current practice patterns of VS in Italy through an anonymous questionnaire addressed to otolaryngologists and neurosurgeons.

\section{Materials and methods}

Study design and survey characteristics

A web-based, anonymous survey was administered to the members of the Italian Society of Otolaryngology, Head and Neck Surgery (SIO) and the Italian Society of Neurosurgery ( $\mathrm{SINCH}$ ) using the Google Drive platform. The survey was conceived in Italian, modifying that administered by the North American Skull Base Society (NASBS) ${ }^{8}$, with permission. The survey was distributed between December 2018 and December 2019; after voluntarily agreeing to participate, surgeons indicated their active involvement into otoneurological/lateral skull base procedures and their surgical specialisation.
The 35-item, multiple-choice survey was divided into three sections: i) general section, investigating years of training, years of experience and volume/year of patients managed and treated; ii) section on overall practice patterns; and iii) section on management options. The full questionnaire is reported in Appendix (https://www.actaitalica.it/article/ view/1107/550).

\section{Statistical analysis}

Categorical variables were presented as frequencies with percentages; data were analysed per item, so the denominator varied per question due to missing data; comparisons between variables were assessed using Chi-square or Fischer's exact tests, as appropriate. All tests were two-tailed and statistical significance was determined by a $p$ value $<0.05$. Data were analysed using the SPSS 20 software (SPSS, IBM Company, Armonk, USA) and GraphPad Prism version 7.00 for Windows, (GraphPad Software, La Jolla California USA, www.graphpad.com).

\section{Results}

Demographics, experience and setting

Sixty-six subjects responded to the survey, nine women $(13.8 \%)$ and 58 men $(86.2 \%)$, mostly within the 50-59 year group of age $(27.3 \%)$. Among them, 37 participants $(56.0 \%), 2$ females $(5.4 \%)$ and 35 males $(94.6 \%)$, confirmed their active involvement in the management of VS, so that their responses were taken into consideration for further analysis. Among the study group involving 25 otolaryngologists $(67.6 \%)$ and $12(32.4 \%)$ neurosurgeons, 30 $(81.1 \%)$ confirmed their specific training in neurotology/ lateral skull base surgery, as shown in Figure 1A. Age distribution and years of experience of the participants are reported in Figures 1B and 1C.

Most respondents, 22 (59.5\%), claimed to work at a university hospital, $12(32.4 \%)$ at hospital without academic affiliation and three $(8.1 \%)$ in private practice. With regards to the volume of cases evaluated by respondents, 17 (46.0\%) managed $\leq 25$ cases/year, while $20(54.0 \%)>25$ cases/ year $(5,13.5 \%$ between 25 and $50 ; 10,27.0 \%$ between 51 and $100 ; 5,13.5 \%>100$ cases/year). There was a significant association between the number of tumours evaluated per year and type of practice setting. Higher volumes of cases ( $>25$ cases/year) were managed at academic settings in $72.7 \%$ of cases while lower volumes $(\leq 25$ cases/year) were mostly treated at hospitals without academic affiliation or in private practice in $73.3 \%$ of cases $(p=0.021)$. No associations were found between type of practice setting and years of experience $(\mathrm{p}=0.279)$ or multidisciplinary approach $(\mathrm{p}=0.317)$. 


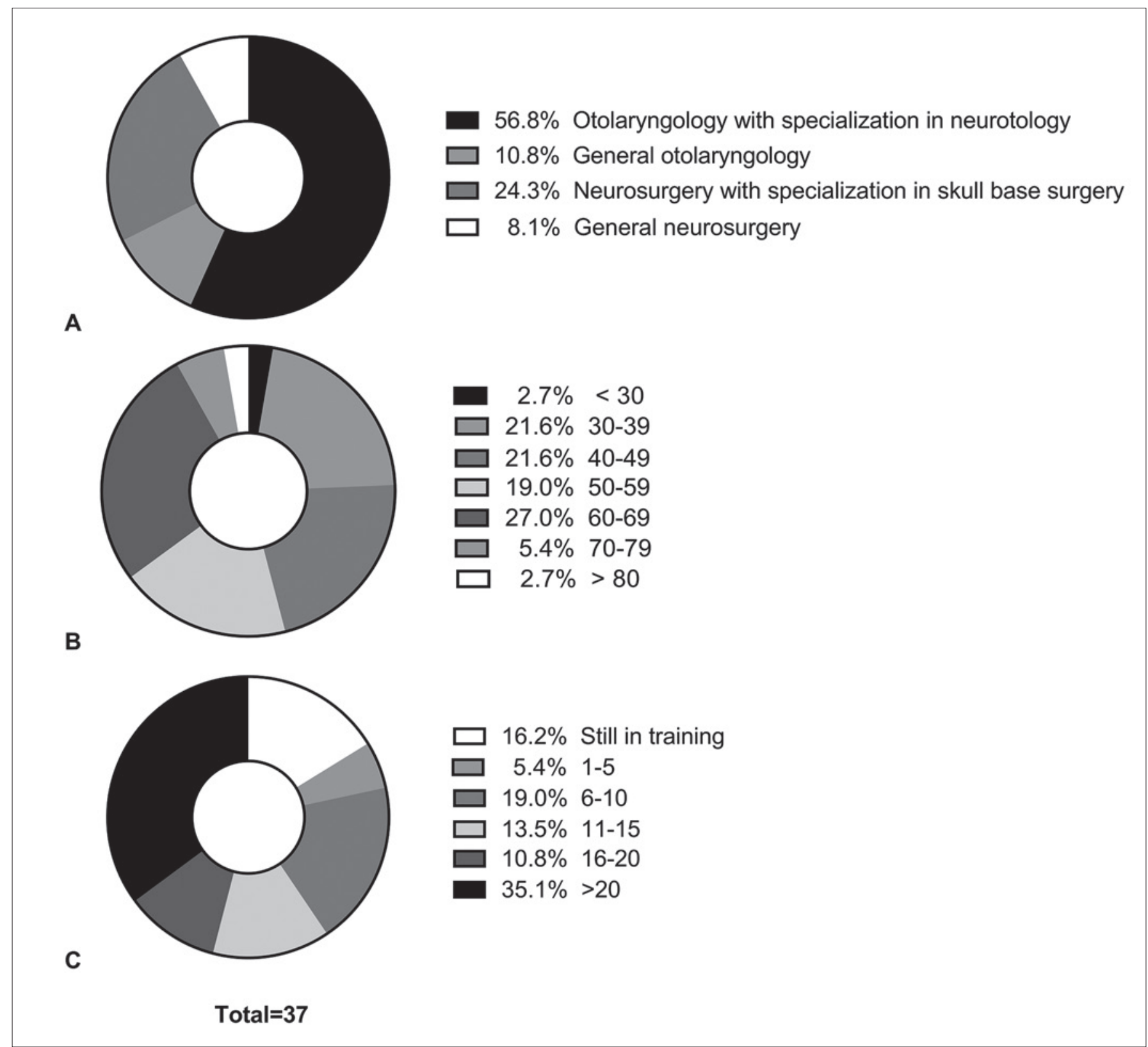

Figure 1. Characteristics of survey participants. (A) Specialisation. (B) Age distribution defined in years. (C) Years of experience in VS care.

\section{Management strategies}

Eighteen respondents $(50.0 \%)$ claimed to discuss patients diagnosed with VS in multidisciplinary meetings. Twentythree $(62.2 \%)$ claimed to surgically treat VS in teams where otolaryngologists and neurosurgeons are involved, and 14 (37.8\%) performed surgical interventions on their own.

\section{a. Intracanalicular VS and tumours $\leq 1.5 \mathrm{~cm}$}

in the cerebello-pontine angle

In case of small VS, 30 respondents $(81.1 \%)$ declared to prefer observation as first option, $4(10.8 \%)$ recommended microsurgical up-front treatment and three $(8.1 \%)$ radiotherapy. Considering tumour observation, the 5-year growth probability for intracanalicular VS and for small, extrameatal VS $(\leq 1.5) \mathrm{cm}$ was considered $<25 \%$ by 27 (75.0\%) and $16(45.7 \%)$ participants, respectively. Most respondents $(28,75.6 \%)$ believed that the best chance of retaining serviceable hearing at 10 -year follow-up, is given by conservative approach.

In case of surgery, 30 participants $(83.4 \%)$ declared to 
consider hearing preservation surgery (HPS) as a feasible strategy, while six (16.6\%) do not perform this surgical option, regardless of hearing status. Given favourable tumour characteristics and contralateral normal hearing, pure tone average $(\mathrm{PTA}) \leq 30 \mathrm{~dB}$ and word recognition score (WRS) $\geq 70 \%$ are considered neccessary to attempt HPS by 19 respondents $(51.4 \%)$, while $5(13.5 \%)$ believed that HPS should be tried with any detectable hearing. Among different factors affecting HPS outcomes, tumour size and preoperative hearing were considered to be the strongest predictors of on hearing outcomes by $28(75.6 \%)$ and 19 $(51.3 \%)$ respondents, respectively (Fig. 2). Tumour origin (from the superior or inferior vestibular nerve), presence of cerebrospinal fluid (CSF) fundal cup, patient's age and internal auditory canal enlargement were considered of average importance. Overall, the preferred approach for HPS was the retrosigmoid (RS) in the $61.2 \%$ of cases, for both otolaryngologists and neurosurgeons $(60.0 \%$ and $58.1 \%$ respectively). More than three-fourths of respondents (77.8\%) claimed to perform both the RS and middle cranial fossa approaches, depending on tumour characteristics. The chance of preserving residual hearing after HPS was estimated to be $<40 \%$ by 25 respondents $(67.5 \%)$.

\section{b. Tumours $>1.5 \mathrm{~cm}$ in the cerebello-pontine angle} In the case of tumours $>1.5 \mathrm{~cm}$ in the cerebello-pontine angle or when HPS is not the target, 19 participants (51.4\%) preferred the translabyrinthine (TL) approach, 11 (29.7\%) the RS approach and seven (18.9\%) claimed to use both surgical corridors independently. When stratified according to specialisation of respondents, the TL approach was preferred by otolaryngologists than neurosurgeons $(18,72.0 \%$ vs $1,8.3 \%, \mathrm{p}<0.001)$. On the contrary, the RS corridor was more frequently used by neurosurgeons $(8,66.7 \% \mathrm{vs}$ $3,12.0 \%, \mathrm{p}<0.001)$.

For large VS $(>3 \mathrm{~cm})$, most respondents would perform a single stage surgical resection $(97.3 \%)$, either via TL $(51.4 \%)$ or RS $(43.2 \%)$ corridors. There was a significant difference between otolaryngologists and neurosurgeons concerning the surgical approach for large VS resections. The former claimed to prefer the TL approach in $68.0 \%$ of cases, while the latter a RS approach in $75.0 \%$ of cases $(\mathrm{p}=0.012)$.

Twenty-eight surgeons (75.6\%) would try to achieve a total resection of large VS, unless the tumour is tenaciously adherent to the facial nerve. Six $(16.2 \%)$ would perform a subtotal resection to reach adequate tumour volume for subsequent stereotactic radiosurgery/stereotactic radiation therapy (SRS/SRT), and only three $(8.1 \%)$ would perform a total resection, even if it could place facial nerve function at risk. Only six (16.2\%) respondents claimed to perform sub-total VS resections in large VS very frequently (more than $50 \%$ of cases). Interestingly, when stratified according to the surgical specialisation, neurosurgeons declared to perform subtotal resections more frequently than otolaryngologists would $(\mathrm{p}<0.001)$.

When asked about intraoperative parameters that could threaten facial nerve function, otolaryngologists consid-

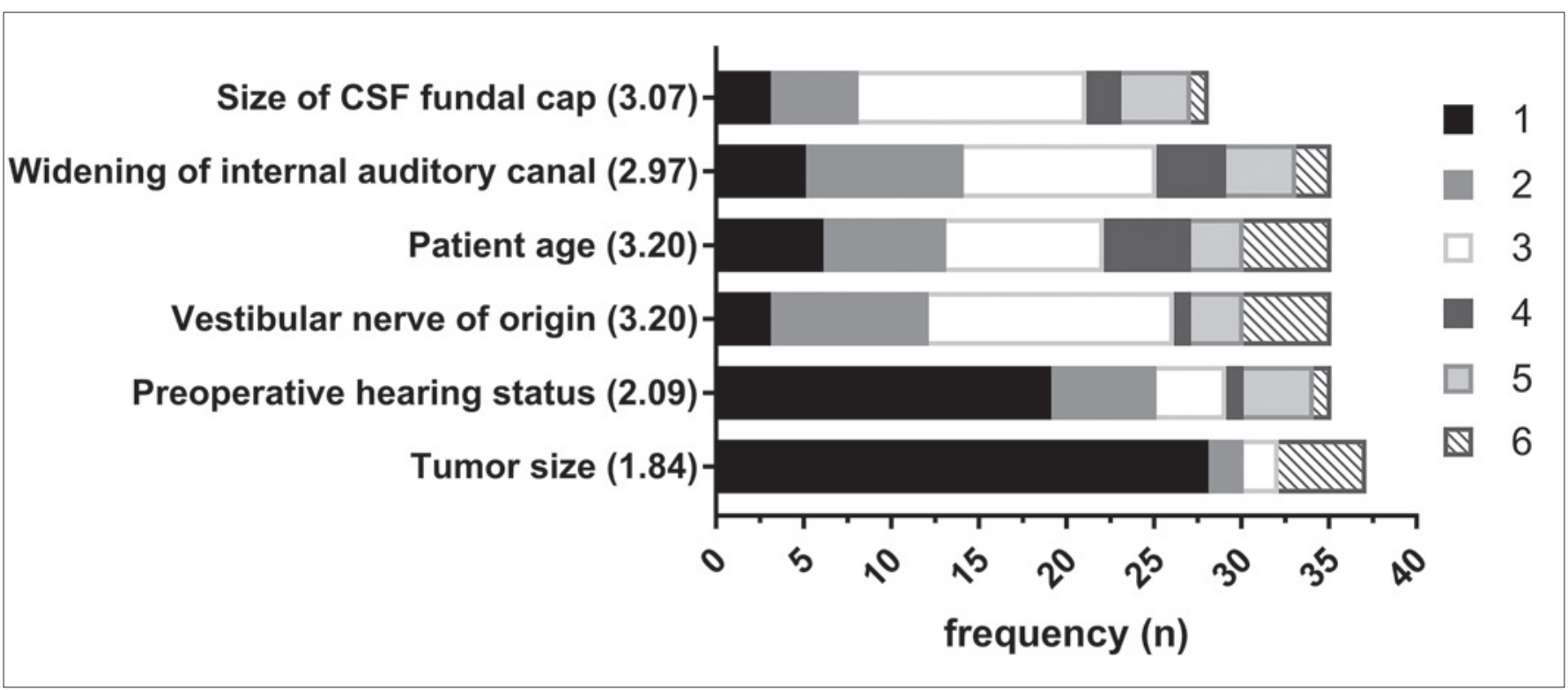

Figure 2. Ranking of predictive factors for hearing preservation weighted by survey participants according to the perceived impact on successful hearing preservation surgery $(1=$ strongest predictor, 6 = weakest predictor). The average rating per item is reported between brackets. 
ered tumour extension and grade of adhesion at the nerve/ tumour interface the most reliable $(18,75.0 \%$ vs $6,25 \%$, $\mathrm{p}=0.044$ ), while neurosurgeons more frequently relied on electrophysiological measures $(6,66.7 \%$ vs $3,33.3 \%$, $\mathrm{p}<0.001$ ). Residual tumours were preferably observed and submitted to SRS/SRT or observed and surgically treated only after documented growth by $26(72.2 \%)$ and seven (19.5\%) respondents, respectively. Early SRS/SRT (within 6 months from surgery) for residual VS was advocated by three $(8.3 \%)$.

\section{Symptom control and complications}

Some questions regarded the surgical effects on symptom control. Most surgeons believe that VS surgical removal has no or unpredictable effects on tinnitus $(12,32.4 \%$, and $20,54.1 \%$, respectively), while only four $(10.8 \%)$ believe that surgical treatment would improve preoperative tinnitus. In case of pre-operative dizziness, 28 (75.7\%) respondents suggested that surgery would lead to reduction/resolution of symptoms.

Dealing with surgical complications, the RS approach was perceived to carry the highest risk of postoperative CSF leak (19 respondents, 51.4\%) by both otolaryngologists and neurosurgeons $(11,44.0 \%$ vs $8,66.4 \%)$. Interestingly, $26(70.3 \%)$ respondents did not avoid the RS approach despite the risk of post-operative headache.

\section{Radiation treatment}

Thirty-one $(83.7 \%)$ respondents recommend SRS/SRT in less than 10 cases/year and most participants believe that radiation treatment is not indicated as a primary option for tumours measuring $>2 \mathrm{~cm}(21.6 \%),>2.5 \mathrm{~cm}(35.2 \%)$, and $>3 \mathrm{~cm}(29.7 \%)$. Malignant transformation of VS after SRS/ SRT is generally considered to be extremely rare by most respondents $(29,78.4 \%)$. The questionnaire was, however, not administered to radiation therapists.

\section{Discussion}

Otolaryngologists and neurosurgeons directly involved in the treatment of VS are faced with a wide range of different management possibilities, ranging from observation to active treatments like surgical tumour removal or radiosurgical irradiation ${ }^{9}$. In the absence of international accepted guidelines in this field, and lacking adequate evidence to guide VS management, the possible variability of practice mainly reflects institutional and/or surgeon's experience biases. In the present study, the current treatment strategies for VS in Italy are reported after a nationwide survey administration among the SIO and SINCH members. Thirty-seven respondents confirmed their active involvement in
VS management, $67.6 \%$ were otolaryngologists and $32.4 \%$ neurosurgeons evaluating in $54.0 \%$ of cases $>25 \mathrm{VS}$ patients/year. In 2006, Goodden et al. ${ }^{10}$ investigated on the adherence to guidelines for diagnosis and treatment of VS in the United Kingdom and Ireland, reporting that 73.0\% of neurosurgeons evaluate patients with otolaryngologists. Similarly, Saeed et al. ${ }^{11}$ obtained comparable results among otolaryngologists. In the present study, with regards to the initial assessment of VS patients, respondents were equally distributed between those who evaluate patients alone $(50.0 \%)$ and those who collaborate in a multidisciplinary team $(50.0 \%)$. Interestingly, considering the multidisciplinary collaboration during surgery, $62.2 \%$ of otolaryngologists and neurosurgeons claimed to work together. Although with higher rates of a multidisciplinary approach to the VS patient, the survey conducted among NASBS members ${ }^{8}$ reflected a similar increase of percentages in multidisciplinary collaboration during the diagnostic $(63.0 \%)$ and surgical phases $(86.0 \%)$ of VS management.

\section{Observation}

The spread of MRI has radically changed the diagnosis and treatment of VS, which today is based at first on observation ${ }^{12}$. Both in Italy and USA ${ }^{8}$ the most widespread strategy for the treatment of small and medium sized VS is initial observation until tumour growth becomes evident. This policy seems to be the best choice for both otolaryngologists and neurosurgeons. In fact, the chance of growth is considered low after 5 years of observation, as shown by both the Italian and NASBS surveys ${ }^{8}$. In this regard, Paldor et al. ${ }^{13}$ conducted a comprehensive review of the literature considering 37 studies on $>4000$ patients and reported that only $50 \%$ of tumours demonstrated a radiologically documented growth after 5 years.

\section{Surgery}

The best surgical approach to treat VS, when hearing preservation is not the goal, is still a matter of debate among lateral skull base surgeons, and is reflected in the present study. A systematic literature review by Hadjipanayis et al. ${ }^{14}$ stated that there is insufficient evidence to support the superiority of either RS or the TL approach for complete VS resection and facial nerve preservation, when serviceable hearing is not present.

In case of small to medium sized tumours, in the NASBS study ${ }^{8}$ the TL approach was preferred, while in Italy a significant divergence between otolaryngologists and neurosurgeons came to notice. In fact, while the former preferred the TL approach (72.0\%), most neurosurgeons claimed to use the RS approach (66.7\%). For large VSs (>3 cm), most North American surgeons claimed to prefer the RS ap- 
proach $(52.6 \%)^{8}$, which is confirmed in Italy only among neurosurgeons $(75.0 \%)$, as otolaryngologists preferred the TL approach (68.0\%).

Facial nerve injury is one of the most frequent complications of VS surgery, particularly when the tumour reaches considerable size ${ }^{15}$. Similar to what was reported in the NASBS study ${ }^{8}$, our survey highlighted that most surgeons $(75.6 \%)$ try to achieve total resection of the tumour mass unless it is tenaciously adherent to the facial nerve. Of note, most neurosurgeons claimed to perform subtotal resections and to rely on electrophysiological measures to demonsrate facial nerve integrity significantly more frequently than otolaryngologists, who were more prone to total resections and to rely on intraoperative assessment of tumour extension and adhesion at the nerve/tumour interface.

Considering the management of residual tumour after subtotal resections, most of the respondents $(70.2 \%)$ would adopt an observation policy followed by SRS/SRT only after documented growth. This is in accordance with Taha et al. ${ }^{16}$ reporting that, in case of important adherence with the facial nerve, a near-total tumour resection with eventual subsequent radiation therapy would be the best strategy to ensure the integrity of the nerve.

Considering symptom control, most respondents held that surgery has an unpredictable effect on tinnitus. Noteworthy, Wang et al. ${ }^{17}$ showed that microsurgical VS resection led to Tinnitus Handicap Index reduction in up to $77 \%$ of cases. Most surgeons in Italy and in North America considered that VS surgical removal led to a reduction or even to the resolution of preoperative dizziness. Similarly, microsurgical tumour removal and trigeminal nerve decompression is considered the preferred treatment in cases with VS and associated trigeminal neuralgia ${ }^{18}$.

\section{Hearing preservation surgery}

Both in Italy and in North America ${ }^{8}$ in case of small tumours most of the respondents agreed that the highest chance to preserve hearing is observation. Reznitsky and Cayé-Thomasen ${ }^{19}$ showed in a systematic review of the literature that the chance of preserving good hearing (PTA $<30 \mathrm{~dB}$ and WRS $>70 \%$ ) after 5 years of observation is $50 \%$. In case of HPS, according to the "Congress of Neurological Surgeons" ${ }^{20}$, the overall probability of maintaining serviceable hearing following microsurgical resection of small to medium-sized sporadic VS is moderately low (> 25\%-50\%), while in case of a good pre-operative hearing level (PTA $<30 \mathrm{~dB}$ and WRS $>70 \%$ ) the overall probability is moderately high $(>50 \%-75 \%)$ after surgery and progressively decreases over time.

HPS is performed by $83.4 \%$ of the Italian survey respondents. While the North American surgeons ${ }^{8}$ preferred the middle cranial fossa approach (43.9\%), Italians are more prone to use the RS corridor for HPS (61.2\%). More than half of respondents, $(54.1 \%)$ considered hearing class A according to the American Academy of Otolaryngology-Head and Neck Surgery (AAO-HNS) classification the main selection criterium for HPS, while most North American surgeons $(63.2 \%)$ considered more permissive preoperative hearing intervals as AAO-HNS hearing classes A and $\mathrm{B}$ ( $\mathrm{PTA} \leq 50$ $\mathrm{dB}$ and SDS $\geq 50 \%$ ) to perform HPS ${ }^{8}$. There was agreement in considering both tumour size and pre-operative hearing levels to be the most important factors influencing HPS outcomes, as resulting from the Italian and American surveys ${ }^{8}$. The recent literature demonstrated that besides the abovementioned predictors of hearing outcomes after surgical VS removal, the presence of a fundal cap at the fundus of the internal auditory canal must also be considered relevant ${ }^{20}$. Furthermore, Zanoletti et al. ${ }^{21}$ investigated the predictors of postoperative hearing in HPS performed through a microscopic RS approach combined with a retrolabyrinthine meatotomy. In the attempt to better define the HPS inclusion criteria for achieving best hearing outcomes, cutoff levels for tumour size and preoperative hearing were calculated.

\section{Complications}

CFS leak is one of the most frequent complications of VS surgery, after VII cranial nerve palsy ${ }^{22}$. While in North America ${ }^{8} \mathrm{TL}$ surgery was considered the approach with a higher risk of postoperative CSF leak (57.9\% of respondents), in Italy both otolaryngologists and neurosurgeons considered the RS approach riskier than the TL (51.4\%). A systematic review of complications conducted in 2012 found the same conclusions, demonstrating that the RS approach was significantly associated with higher risk of CSF leak than RS and middle cranial fossa approach ${ }^{22}$.

Post-operative headache is commonly observed after the RS approach ${ }^{22,23}$, although $70.3 \%$ of Italian and $71.6 \%$ of North American surgeons ${ }^{8}$ did not consider this event an absolute contraindication for the approach.

\section{Radiation}

Radiation treatment of VS is rarely proposed in Italy by either otolaryngologists or neurosurgeons; $83.7 \%$ of the interviewees recommend SRS/SRT in less than 10 cases/year. Regarding the cut-off size of tumours to address for SRS/ SRT, there was a substantial orientation among respondents in both Italy and in North America to consider tumours between 2 and $3 \mathrm{~cm}$ as candidates for radiation treatment, excluding those with larger (for which the first option remains surgical) and smaller dimensions (for which the best strategy is observation). A study conducted by Golfinos et al. ${ }^{24}$ showed that SRS for tumours $\leq 2.8 \mathrm{~cm}$ was associated 
with low morbidity and good response to treatment. One of the drawbacks of radiation therapy, i.e the possibility of malignant degeneration after treatment ${ }^{25}$, is considered in both the NASBS and the present studies as an infrequent event.

\section{Conclusions}

The present study documents current practice patterns of VS care in Italy and provides the first overview on VS management of the nationwide community of otoneurosurgical, neurosurgical and skull base surgeons. Although adopted by most centres for surgical VS treatment, a multidisciplinary model of care including otolaryngologists and neurosurgeons needs to be encouraged, especially regarding the initial VS assessment and decision-making. Tumour observation and radiological growth monitoring is advocated by most interviewees in case of small VS. However, there is wide heterogeneity in experience and practices for VS care, mostly influenced by the surgeon's different specialties. These results reflect a general lack of scientific evidence, and national guidelines will foster future research in this field.

\section{References}

1 Tos M, Stangerup S, Cayé-Thomasen P, et al. What is the real incidence of vestibular schwannoma? Arch Otolaryngol Head Neck Surg 2004;130:216-220. https://doi.org/10.1001/archotol.130.2.216

2 Sandifort E. De duram quodam corpusculo, nervo auditorio adherente. In: Van de Eyk P, Vygh D, editors. Observationes Anatomico-Pathologicae, Book 1. 1777. pp.116-120.

3 Moffat D, Quaranta N, Baguley D, et al. Management strategies in neurofibromatosis type 2. Eur Arch Otorhinolaryngol 2003;260:1218. https://doi.org/10.1007/s00405-002-0503-9

4 Moffat D, Baguley D, Von Blumenthal H, et al. Sudden deafness in vestibular schwannoma. J Laryngol Otol 1994;108:116-119. https:// doi.org/10.1017/S0022215100126052

5 Reznitsky M, Petersen MMBS, West N, et al. Epidemiology of vestibular schwannomas - prospective 40-year data from an unselected national cohort. Clin Epidemiol 2019;11:981-986. https://doi. org/10.2147/CLEP.S218670

6 Marinelli JP, Grossardt BR, Lohse CM, et al. Prevalence of sporadic vestibular schwannoma: reconciling temporal bone, radiologic, and population-based studies. Otol Neurotol 2019;40:384-390. https:// doi.org/10.1097/MAO.0000000000002110

7 Daveau C, Zaouche S, Jouanneau E, et al. Experience of multidisciplinary team meetings in vestibular schwannoma: a preliminary report. Eur Arch Otorhinolaryngol 2015;272:3187-192. https://doi. org/10.1007/s00405-014-3375-x

8 Carlson ML, Van Gompel JJ, Wiet RM, et al. A cross-sectional survey of the North American Skull Base Society: current practice patterns of vestibular schwannoma evaluation and management in North America. J Neurol Surg B Skull Base 2018;79:289-296. https://doi. org/10.1055/s-0037-1607319

9 Zanoletti E, Mazzoni A, Martini A, et al. Surgery of the lateral skull base: a 50-year endeavour. Acta Otorhinolaryngol Ital 2019;39(Suppl. 1):S1-S146. https://doi.org/10.14639/0392-100X-suppl.1-39-2019
10 Goodden JR, Tranter R, Hardwidge C. Setting the standard - UK neurosurgical acoustic neuroma practice. Ann R Coll Surg Engl 2006;88:486-489. https://doi.org/10.1308/003588406X114893

11 Saeed SR, Suryanarayanan R, Dezso A, et al. Vestibular schwannoma management: current practice amongst UK otolaryngologists - time for a national prospective audit. Ann R Coll Surg Engl 2006;88:490495. https://doi.org/10.1308/003588406X114901

12 Yao L, Alahmari M, Temel Y, et al. Therapy of sporadic and NF2related vestibular schwannoma. Cancers (Basel) 2020;12:835. https:// doi.org/10.3390/cancers12040835

13 Paldor I, Chen AS, Kaye AH. Growth rate of vestibular schwannoma. J Clin Neurosci 2016;32:1-8. https://doi.org/10.1016/j. jocn.2016.05.003

14 Hadjipanayis CG, Carlson ML, Link MJ, et al. Congress of Neurological Surgeons Systematic Review and Evidence-Based Guidelines on surgical resection for the treatment of patients with vestibular schwannomas. Neurosurgery 2018;82:E40-E43. https://doi.org/10.1093/neuros/nyx 512

15 Grinblat G, Dandinarasaiah M, Braverman I, et al. Large and giant vestibular schwannomas: overall outcomes and the factors influencing facial nerve function. Neurosurg Rev 2020 Aug 29 (Epub ahead of print). https://doi.org/10.1007/s10143-020-01380-6

16 Taha I, Hyvärinen A, Ranta A, et al. Facial nerve function and hearing after microsurgical removal of sporadic vestibular schwannomas in a population-based cohort. Acta Neurochir (Wien) 2020;162:43-54. https://doi.org/10.1007/s00701-019-04055-4

17 Wang JJ, Feng YM, Wang H, et al. Changes in tinnitus after vestibular schwannoma surgery. Sci Rep 2019;9:1743. https://doi.org/10.1038/ s41598-019-38582-y

18 Elahi F, Ho KW. Anesthesia dolorosa of trigeminal nerve, a rare complication of acoustic neuroma surgery. Case Rep Neurol Med 2014;2014:496794. https://doi.org/10.1155/2014/496794

19 Reznitsky M, Cayé-Thomasen P. Systematic review of hearing preservation in observed vestibular schwannoma. J Neurol Surg B Skull Base 2019;80:165-168. https://doi.org/10.1055/s-0039-1679894

20 Carlson ML, Vivas EX, McCracken DJ, et al. Congress of Neurological Surgeons Systematic Review and Evidence-Based Guidelines on hearing preservation outcomes in patients with sporadic vestibular schwannomas. Neurosurgery 2018;82:E35-E39. https://doi. org/10.1093/neuros/nyx511

21 Zanoletti E, Mazzoni A, Frigo AC, et al. Hearing preservation outcomes and prognostic factors in acoustic neuroma surgery: predicting cutoffs. Otol Neurotol 2020;41:686-693. https://doi.org/10.1097/ MAO.0000000000002602

22 Ansari SF, Terry C, Cohen-Gadol AA. Surgery for vestibular schwannomas: a systematic review of complications by approach. Neurosurg Focus 2012;33:E14. https://doi.org/10.3171/2012.6.FOCUS12163

23 Sabab A, Sandhu J, Bacchi S, et al. Postoperative headache following treatment of vestibular schwannoma: a literature review. J Clin Neurosci 2018;52:26-31. https://doi.org/10.1016/j.jocn.2018.04.003

24 Golfinos JG, Hill TC, Rokosh R, et al. A matched cohort comparison of clinical outcomes following microsurgical resection or stereotactic radiosurgery for patients with small- and medium-sized vestibular schwannomas. J Neurosurg 2016;125:1472-1482. https://doi. org/10.3171/2015.12.JNS151857

25 Germano IM, Sheehan J, Parish J, et al. Congress of Neurological Surgeons Systematic Review and Evidence-Based Guidelines on the role of radiosurgery and radiation therapy in the management of patients with vestibular schwannomas. Neurosurgery 2018;82:E49-E51. https://doi.org/10.1093/neuros/nyx515 